\title{
Magnetogravitodynamic stability of streaming fluid cylinder under the effect of capillary force
}

\author{
Alfaisal A Hasan ${ }^{1 *}$ and Rasha A Abdelkhalek²
}

\section{"Correspondence:}

alfaisal772001@yahoo.com 'Basic and Applied Sciences

Department, College of Engineering and Technology, Arab Academy for Science \& Technology and Maritime Transport (AASTMT), Elhorria, P.O. Box 2033, Cairo, Egypt Full list of author information is available at the end of the article

\begin{abstract}
The magnetohydrodynamic stability criterion of self-gravitating streaming fluid cylinder under the combined effect of self-gravitating, magnetic, and capillary forces has been derived. The results are discussed analytically and some data are verified numerically for different parameters of the problem. The magnetic and capillary forces are stabilizing, but the streaming is destabilizing while the self-gravitating is stabilizing or destabilizing according to restrictions. The stable and unstable domains are identified and, moreover, the influences of the magnetic and capillary forces on the self-gravitating instability of the model have been examined. Including the magnetic force together with self-gravitating force improves the instability of the model. However, the self-gravitating instability will never be suppressed whatever the effects of the MHD force stabilizing effects are.
\end{abstract}

Keywords: self-gravitating; magnetohydrodynamic; capillary; streaming

\section{Introduction}

The stability of a fluid cylinder under the action of the capillary or/and other forces has received the attention of several researchers (Rayleigh [1], Yuen [2], Nayfeh and Hassan [3] and Kakutani et al. [4]. The effect of the electromagnetic Lorentz force on the capillary instability has been examined in several texts by the Nobel prize winner (1986) Chandrasekhar [5]. This has been done only for small axisymmetric perturbation and with a constant magnetic field. Radwan et al. [6-10] extended such interesting works by studying the magnetohydrodynamic stability of a liquid jet embedded into a tenuous medium for all axisymmetric and non-axisymmetric modes of perturbation. The stability of different cylindrical models under the action of self-gravitating force in addition to other forces has been elaborated by Radwan and Hasan [9] and [10]. They [9] studied the gravitational stability of a fluid cylinder under transverse time-dependent electric field for axisymmetric perturbations. Hasan [11] discussed the stability of oscillating streaming fluid cylinder subject to the combined effect of the capillary, self-gravitating, and electrodynamic forces for all axisymmetric and non-axisymmetric perturbation modes. He [12] studied the instability of a full fluid cylinder surrounded by self-gravitating tenuous medium pervaded by transverse varying electric field under the combined effect of the capillary, self-gravitating, and electric forces for all modes of perturbations. In [13] Hasan et al. investigated the hydromagntic stability of a self-gravitational oscillating streaming fluid jet pervaded by az-

(c) 2013 Hasan and Abdelkhalek; licensee Springer. This is an Open Access article distributed under the terms of the Creative Commons Attribution License (http://creativecommons.org/licenses/by/2.0), which permits unrestricted use, distribution, and reproduction in any medium, provided the original work is properly cited. 
imuthal varying magnetic field for all axisymmetric and non-axisymmetric modes of perturbation. He [14] discussed the stability of oscillating streaming self-gravitating dielectric incompressible fluid cylinder surrounded by tenuous medium of negligible motion pervaded by transverse varying electric field for all modes of perturbations. He [15] studied the magnetodynamic stability of a fluid jet pervaded by a transverse varying magnetic field while its surrounding tenuous medium is penetrated by uniform magnetic field.

The present work is devoted to studying the magnetogravitodynamic stability of a streaming fluid cylinder and examining the influence of capillary and magnetic forces on the self-gravitating instability of the present models. This may be carried out, for all axisymmetric and non-axisymmetric modes of perturbation, analytically and the results will be verified numerically.

\section{Formulation of the problem}

We consider a uniform cylinder of an incompressible inviscid fluid of radius $R_{0}$ surrounded by a tenuous medium of negligible motion. In the initial unperturbed state, the model is assumed to be streaming uniformly with velocities

$$
\underline{u}_{0}=(0, W, U)
$$

and pervaded internally and externally by the magnetic fields

$$
\underline{H}_{0}=\left(0,0, H_{0}\right), \quad \underline{H}_{0}^{e x}=\left(0,0, \alpha H_{0}\right) .
$$

Here $W$ and $U$ are (constants) the speed of the fluid, $H_{0}$ is the intensity of the magnetic field in the fluid, and $\alpha$ is some parameter. The components of $u_{0}, H_{0}, H_{0}^{e x}$ are considered along the cylindrical coordinates $(r, \varphi, z)$ with the $z$-axis coinciding with the axis of the cylinder as shown in Figure 1. The fluid matter of the cylinder is acted upon by the combined effects of the self-gravitating, inertial, capillary, and magnetic forces. The surrounding tenuous medium of the fluid cylinder is acted upon by the self-gravitating and magnetic forces only.

Figure 1 Sketch for a gravitational MHD fluid cylinder.

$$
\underline{H}_{0}=\left(0,0, H_{0}\right)
$$

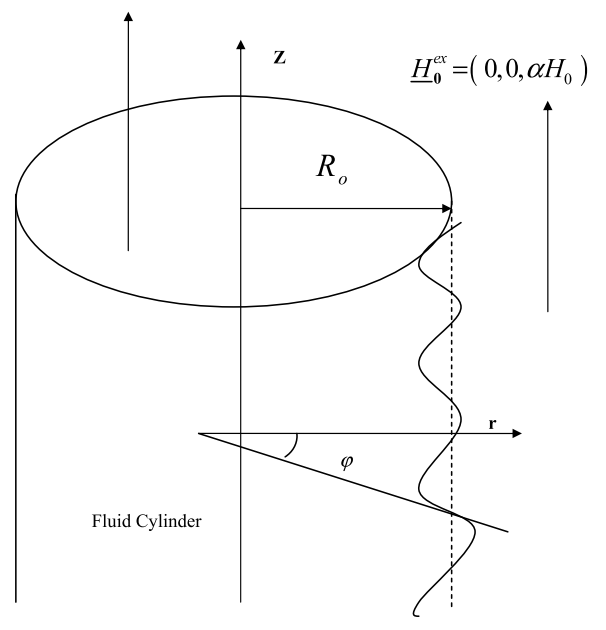


The required basic equations for such kind of study may be obtained by combining the ordinary hydrodynamic equations and those of Maxwell's concerning the electromagnetic field theory together with Newtonian gravitational field equations.

For the problem at hand, under the present circumstances, these equations are the following.

For the fluid, we have

$$
\begin{aligned}
& \rho\left[\frac{\partial \underline{u}}{\partial t}+(\underline{u} \cdot \nabla \underline{u})\right]=-\nabla P+\rho \nabla V+\left(\frac{\mu}{4 \pi}\right)(\nabla \wedge \underline{H}) \wedge \underline{H}, \\
& \nabla \cdot \underline{u}=0, \\
& \nabla \cdot \underline{H}=0, \\
& \frac{\partial \underline{H}}{\partial t}=\nabla \wedge(\underline{u} \wedge \underline{H}), \\
& \nabla^{2} V=-4 \pi \rho G .
\end{aligned}
$$

The curvature pressure due to the capillary force is

$$
P_{s}=T\left(\nabla \cdot \underline{N}_{s}\right)
$$

with

$$
\underline{N}_{s}=\nabla F /|\nabla F|,
$$

where

$$
F(r, \varphi, z)=0
$$

is the boundary surface equation at time $t$, while $\underline{N}_{s}$ is a unit outward vector normal to the surface, $T$ is surface tension, and $P_{s}$ is pressure due to curvature.

For the surrounding tenuous medium, the basic equations are

$$
\begin{aligned}
& \nabla \cdot \underline{H}^{e x}=0, \\
& \nabla \wedge \underline{H}^{e x}=0, \\
& \nabla^{2} V^{e x}=0 .
\end{aligned}
$$

Here $\rho, u_{0}$, and $P$ are the fluid mass density, velocity vector, and kinetic pressure, respectively; $H_{0}, H_{0}^{e x}$ are the magnetic field intensities and $V, V^{e x}$ are self-gravitating potentials, respectively, inside and outside the fluid cylinder, $\mu$ is the magnetic field permeability coefficient and $G$ is the gravitational constant.

\section{Unperturbed state}

The unperturbed state is studied and the fundamental quantities of such state could be obtained. Equation (1) together with equation (3) gives

$$
\rho \nabla V_{0}-\nabla P_{0}+\left(\frac{\mu}{4 \pi}\right)\left(\underline{H}_{0} \cdot \nabla\right) \underline{H}_{0}-\left(\frac{\mu}{8 \pi}\right) \nabla\left(\underline{H}_{0} \cdot \underline{H}_{0}\right)=0,
$$

from which, taking into account equation (5), we obtain $\nabla\left(\rho V_{0}-P_{0}-\left(\frac{\mu}{8 \pi}\right) H_{0}^{2}\right)=0$. 
By integrating this equation, we get

$$
P_{0}=\rho V_{0}-\left(\frac{\mu}{8 \pi}\right) H_{0}^{2}+C,
$$

where $C$ is a constant of integration to be determined.

The surface pressure due to the capillary force ( $c f$. Chandrasekhar [5]) is given by

$$
P_{0 s}=T / R_{0}
$$

The self-gravitating potentials $V_{0}$ and $V_{0}^{e x}$ of the unperturbed state satisfy

$$
\begin{aligned}
& \nabla^{2} V_{0}^{e x}=-4 \pi \rho G, \\
& \nabla^{2} V_{0}^{e x}=0 .
\end{aligned}
$$

The non-singular solutions of equations (17) and (18) in the cylindrical coordinates $(r, \varphi, z)$ with cylindrical symmetries $\left(\frac{\partial}{\partial \varphi}\right)=0$ and $\left(\frac{\partial}{\partial z}\right)=0$ are given by

$$
\begin{aligned}
& V_{0}=-\pi G \rho r^{2}+C_{1}, \\
& V_{0}^{e x}=C_{2} \ln r+C_{3},
\end{aligned}
$$

where $C_{1}, C_{2}$, and $C_{3}$ are constants of integration to be determined. By applying the conditions that the self-gravitational potential $V$ and its derivative must be continuous across the unperturbed boundary surface at $r=R_{0}$ and choosing $C_{1}=0$ since the potential inside the cylinder is zero, we get

$$
\begin{aligned}
& C_{2}=-2 \pi G \rho R_{0}^{2}, \\
& C_{3}=-\pi G \rho R_{0}^{2}+2 \pi G \rho R_{0}^{2} \ln r .
\end{aligned}
$$

Therefore,

$$
\begin{aligned}
& V_{0}=-\pi G \rho R_{0}^{2}, \\
& V_{0}^{e x}=-\pi G \rho R_{0}^{2}\left(1+2 \ln \left(r / R_{0}\right)\right) .
\end{aligned}
$$

Moreover, by applying the condition that the total pressure must be balanced across the boundary surface at $r=R_{0}$, the distribution of the fluid pressure in the unperturbed state is given by

$$
P_{0}=\left(\frac{T}{R_{0}}\right)+\pi G \rho^{2}\left(R_{0}^{2}-r^{2}\right)+\left(\frac{\mu}{8 \pi}\right) H_{0}^{2}\left(\alpha^{2}-1\right) .
$$

It is worth noting that in the absence of surface tension at the boundary surface

$$
\alpha \geq 0
$$

in order that

$$
P \geq 0 \text {. }
$$




\section{Perturbation analysis}

We consider small departures from an unperturbed right-cylindrical shape of an incompressible fluid. Therefore a normal mode can be expressed uniquely in terms of the deformed surface. Hence we may assume that the deformed interface is described by

$$
r=R_{0}+\varepsilon(t) R_{1}+\cdots
$$

with

$$
R_{1}=\exp (i(k z+m \varphi))
$$

Here $R_{1}$ is the elevation of the surface wave measured from the unperturbed position, $k$ (real number) is the longitudinal wave number, $m$ (integer) is the transverse wave number. The amplitude $\varepsilon(t)$ of the perturbation is given by

$$
\varepsilon(t)=\varepsilon_{0} \exp (\sigma t)
$$

where $\varepsilon_{0}(=\varepsilon$ at $t=0)$ is the initial amplitude and $\sigma$ is the temporal amplification. If $\sigma(=i \omega$, $i=\sqrt{-1}$ ) is imaginary, then $\omega / 2 \pi$ is the oscillation frequency of the propagating wave in the fluid.

As the initial streaming state is perturbed, every physical quantity $Q(r, \varphi, z ; t)$ may be expanded as

$$
Q(r, \varphi, z, t)=Q_{0}(r)+Q_{1}(r, \varphi, z, t) .
$$

Here $Q$ stands for $P, u, V, V^{e x}, H, H^{e x}$, and $N_{s}$ while $Q_{0}$ indicates the unperturbed quantity and $Q_{1}$ is a small increment of $Q$ due to disturbances.

In view of the expansion (31), the basic equations of motion (3)-(13) in the perturbation state give

$$
\begin{aligned}
& \rho\left(\frac{\partial \underline{u}_{1}}{\partial t}+\left(\underline{u}_{0} \cdot \nabla \underline{u}_{1}\right)\right)=-\nabla P_{1}+\rho \nabla V_{1}+\left(\frac{\mu}{4 \pi}\right)\left(\underline{H}_{0} \cdot \nabla\right) \underline{H}_{1} \\
& -\left(\frac{\mu}{8 \pi}\right) \nabla\left(2 \underline{H}_{0} \cdot \underline{H}_{1}\right), \\
& \nabla \cdot \underline{u}_{1}=0, \\
& \nabla \cdot \underline{H}_{1}=0, \\
& \left(\frac{\partial \underline{H}_{1}}{\partial t}\right)=\left(\underline{H}_{0} \cdot \nabla\right) \underline{u}_{1}-\left(\underline{u}_{0} \cdot \nabla\right) \underline{H}_{1}, \\
& \nabla^{2} V_{1}=0 \text {, } \\
& P_{1 s}=\left(\frac{-T}{R_{0}^{2}}\right)\left[R_{1}+\left(\frac{\partial^{2} R_{1}}{\partial \varphi^{2}}\right)+R_{0}^{2}\left(\frac{\partial^{2} R_{1}}{\partial z^{2}}\right)\right] \text {, } \\
& \nabla \cdot \underline{H}_{1}^{e x}=0 \text {, } \\
& \nabla \wedge \underline{H}_{1}^{e x}=0, \\
& \nabla^{2} V_{1}^{e x}=0 \text {, }
\end{aligned}
$$


where equations (33) and (34) have been used to obtain equation (35). Based on the linear perturbation technique, the linearized quantity $Q_{1}(r, \varphi, z ; t)$ may be expressed as

$$
Q_{1}(r, \varphi, z ; t)=\varepsilon_{0} q_{1}(r) \exp (\sigma t+i(k z+m \varphi))
$$

By means of the expansion (41), equations (36) and (40) give the second-order ordinary differential equation

$$
\left(\frac{1}{r}\right)\left(\frac{d}{d r}\right)\left(r\left(\frac{d \phi_{1}(r)}{d r}\right)\right)-\left(\left(\frac{m^{2}}{r^{2}}\right)+k^{2}\right) \phi_{1}(r)=0
$$

where $\phi_{1}(r)$ stands for $V_{1}(r)$ and $V_{1}^{e x}(r)$. The solution of equation (42) is given in terms of the ordinary Bessel functions of imaginary argument. For the problem under consideration, apart from the singular solution, the solutions of equations (36) and (40) are finally given by

$$
\begin{aligned}
& V_{1}=\varepsilon_{0} A I_{m}(k r) \exp (\sigma t+i(k z+m \varphi)), \\
& V_{1}^{e x}=\varepsilon_{0} B K_{m}(k r) \exp (\sigma t+i(k z+m \varphi)) .
\end{aligned}
$$

Here $I_{m}(k r)$ and $K_{m}(k r)$ are the modified Bessel functions of the first and second kind of order $m$, while $A$ and $B$ are constants of integration to be determined.

Using the space-time dependence (41) for equation (32), we get

$$
(\sigma+i m W+i k U) \underline{u_{1}}-(i \mu k / 4 \pi \rho) H_{0} \underline{H_{1}}=-\nabla \Pi_{1}
$$

with

$$
\Pi_{1}=\left(\frac{P_{1}}{\rho}\right)-V_{1}+(\mu / 4 \pi \rho)\left(\underline{H}_{0} \cdot \underline{H}_{1}\right) .
$$

Also, equation (35) yields

$$
\underline{H}_{1}=\left(\frac{i k H_{0}}{\sigma+i m W+i k U}\right) \underline{u_{1}} .
$$

By combining equations (45) and (47), we get

$$
\underline{u_{1}}=\left(\frac{-(\sigma+i m W+i k U)}{\left((\sigma+i m W+i k U)^{2}+\Omega_{A}^{2}\right)}\right) \nabla \Pi_{1},
$$

where

$$
\Omega_{A}=\left(\frac{\mu k^{2} H_{0}^{2}}{4 \pi \rho}\right)^{1 / 2}
$$

is the Alfven wave frequency defined in terms of $H_{0}$.

By taking the divergence of both sides of equation (48) and using equation (33), we obtain

$$
\nabla^{2} \Pi_{1}=0 .
$$


Using the space dependence (41) for equation (50) and following similar steps for the resulting differential equation as has already been done for equations (36) and (40), the solution of equation (50) could be obtained. Therefore, the non-singular solution for $\Pi_{1}(r, \varphi, z ; t)$ is given by

$$
\Pi_{1}=C_{4} \varepsilon_{0} I_{m}(k r) \exp (\sigma t+i(k z+m \varphi))
$$

where $C_{4}$ is a constant of integration to be determined.

The pressure surface $P_{1 s}$ in the perturbed state due to the capillary force is determined from equation (37) along with (29) in the form

$$
P_{1 s}=\left(\frac{-T}{R_{0}^{2}}\right)\left(1-m^{2}-x^{2}\right) \exp (\sigma t+i(k z+m \varphi))
$$

where $x\left(=k R_{0}\right)$ is the dimensionless longitudinal wavenumber.

Now, equation (34) means that the magnetic field intensity $H_{1}^{e x}$ in the perturbed state may be derived from a scalar function, $\psi_{1}^{e x}$ say, such that

$$
H_{1}^{e x}=\nabla \psi_{1}^{e x}
$$

By combining equations (38) and (53), we get

$$
\nabla^{2} \psi_{1}^{e x}=0
$$

Similarly, as it has been done for equation (50), equation (54) is solved and its finite solution is given by

$$
\psi_{1}^{e x}=C_{5} \varepsilon_{0} K_{m}(k r) \exp (\sigma t+i(k z+m \varphi))
$$

where $C_{5}$ is a constant of integration to be determined upon applying boundary conditions.

\section{Boundary conditions}

The solution of the basic equations (3)-(13) in the unperturbed state given by (23)-(25) together with (1), (2) and (6) and in the perturbed state given by (43)-(55) must satisfy appropriate boundary conditions. These boundary conditions must be applied across the perturbed interface (28) at the unperturbed boundary surface $r=R_{0}$.

Under the present circumstances, these boundary conditions may be stated as follows.

(i) Self-gravitating conditions.

The gravitational potential and its derivative must be continuous across the perturbed fluid interface (28) at the unperturbed boundary $r=R_{0}$. These conditions at $r=R_{0}$ read

$$
\begin{aligned}
& V_{1}+R_{1}\left(\frac{\partial V_{0}}{\partial r}\right)=V_{1}^{e x}+R_{1}\left(\frac{\partial V_{0}^{e x}}{\partial r}\right), \\
& \left(\frac{\partial V_{1}}{\partial r}\right)+R_{1}\left(\frac{\partial^{2} V_{0}}{\partial r^{2}}\right)=\left(\frac{\partial V_{1}^{e x}}{\partial r}\right)+R_{1}\left(\frac{\partial^{2} V_{0}^{e x}}{\partial r^{2}}\right) .
\end{aligned}
$$


By substituting from equations (23), (24), (29), (43) and (44) into the conditions (56) and (57), we get

$$
\begin{aligned}
& A I_{m}(x)=B K_{m}(x), \\
& A k I_{m}^{\prime}(x)=B k K_{m}^{\prime}(x)+4 \pi G \rho,
\end{aligned}
$$

from which we obtain

$$
\begin{aligned}
& A=4 \pi \rho G R_{0} K_{m}(x), \\
& B=4 \pi \rho G R_{0} I_{m}(x),
\end{aligned}
$$

where $x\left(=k R_{0}\right)$ is the dimensionless longitudinal wave number.

(ii) Kinematic condition.

The normal component of the velocity vector u must be compatible with the velocity of the particles of the boundary surface (28) at the unperturbed surface $r=R_{0}$. This condition reads

$$
\underline{u}_{1 r}=\frac{\partial R_{1}}{\partial t}+U \frac{\partial R_{1}}{\partial z}+W \frac{\partial R_{1}}{\partial \varphi} .
$$

Using equations (29), (48) and (51) for the condition (62), we obtain

$$
C_{4}=\left(-(\sigma+i m W+i k U)^{2}+\Omega_{A}^{2}\right)\left(R_{0} / x I_{m}^{\prime}(x)\right) .
$$

(iii) Magnetodynamic condition.

The jump of the normal component of the magnetic field vanishes across the fluid perturbed interface at $r=R_{0}$. This means that

$$
\underline{N_{s}} \cdot \underline{H}-\underline{N_{s}} \cdot \underline{H}^{e x}=0 \quad \text { at } r=R_{0},
$$

from which we obtain

$$
\underline{H}_{1 r}-\underline{H}_{1 r}^{e x}=i k R_{1} H_{0}(1-\alpha) \text {. }
$$

Therefore, upon using equations (47), (48), (51), (53), and (55) for (65), we get

$$
C_{5}=\frac{i \alpha H_{0}}{K_{m}^{\prime}(x)}
$$

\section{Dispersion relation}

Here we apply a compatibility condition known as the compatibility dynamical condition.

The normal component of the velocity vector $u$ must be compatible with the velocity of the particles of the boundary surface (24) at the unperturbed surface $r=R_{0}$. 
Mathematically, this condition could be given as

$$
P_{1}+R_{1} \frac{\partial P_{0}}{\partial r}+\frac{\mu}{4 \pi}\left(\underline{H}_{0} \cdot \underline{H}_{1}\right)-\frac{\mu}{4 \pi}\left(\underline{H}_{0} \cdot \underline{H}_{1}\right)^{e x}=P_{1 s} .
$$

This may be rewritten, on using equation (46), in the form

$$
\rho\left(\Pi_{1}+V_{1}\right)=P_{1 s}+\frac{\mu}{4 \pi}\left(\underline{H}_{0} \cdot \underline{H}_{1}\right)^{e x}-R_{1} \frac{\partial P_{0}}{\partial r} .
$$

By substituting from equations (2), (25), (29), (43), (51)-(55), (63), (64), and (66) into the condition (68), the following dispersion relation is obtained:

$$
\begin{aligned}
(\sigma+i m W+i k U)^{2}= & 4 \pi G \rho \frac{x I_{m}^{\prime}(x)}{I_{m}(x)}\left[I_{m}(x) K_{m}(x)-\frac{1}{2}\right]+\frac{T}{\rho R_{0}^{3}}\left(1-m^{2}-x^{2}\right) \frac{x I_{m}^{\prime}(x)}{I_{m}(x)} \\
& +\frac{\mu H_{0}^{2}}{\left(4 \pi \rho R_{0}^{2}\right)}\left[-x^{2}+\alpha^{2} \frac{x^{2} I_{m}^{\prime}(x) K_{m}(x)}{I_{m}(x) K_{m}^{\prime}(x)}\right] .
\end{aligned}
$$

\section{Limiting cases}

The relation (69) is the desired stability criterion of a streaming fluid cylinder under the combined effects of the capillary, inertia, self-gravitating, and magnetic forces. It is a linear combination of the dispersion relations of a streaming fluid cylinder under the influence of the self-gravitating force only, fluid cylinder under the effects of the capillary force only and the one under the electromagnetic force only.

It contains the natural quantity $\left(T / \rho R_{0}^{3}\right)^{-\frac{1}{2}}$ as well as $\left(\mu H_{0}^{2} / 4 \pi \rho R_{0}^{2}\right)^{-\frac{1}{2}}$ together with $(4 \pi G \rho)^{-\frac{1}{2}}$, each as a unit of time. In reality the latter quantities are very interesting and have very important task as we intend to rewrite the relation (69) in a dimensionless form because $\sigma$ has a unit of (time) $)^{-1}$. This situation is exactly the same as the following cases of Chandrasekhar [5] which were performed for axisymmetric $(m=0)$ perturbation of nonstreaming fluid cylinder:

$$
\left(T=0, G \neq 0, H_{0} \neq 0\right), \quad\left(T \neq 0, G=0, H_{0} \neq 0\right) \quad \text { and } \quad\left(T \neq 0, G \neq 0, H_{0}=0\right) .
$$

The relation (69) relates the temporal amplification $\sigma$ with the longitudinal wave number $x$; the modified Bessel functions $I_{m}(x)$ and $K_{m}(x)$ of the first and second kind of order $m$ and with their derivatives, the magnetic field parameter $\alpha$, the self-gravitating constant $G$, the basic magnetic field intensity $H_{0}$, the fluid density $\rho$, the radius $R_{0}$ of the cylinder and with the coefficient $\mu$ of the magnetic permeability.

Since the stability criterion (69) is a general relation, we may obtain several published works as limiting cases from it.

Some approximations $\left(\alpha=0, H_{0}=0, U=0, W=0, T=0\right.$ and $\left.m=0\right)$ are required for equation (69) to yield

$$
\sigma^{2}=4 \pi G \rho \frac{x I_{1}(x)}{I_{0}(x)}\left[I_{0}(x) K_{0}(x)-\frac{1}{2}\right], \quad I_{0}^{\prime}(x)=I_{1}(x),
$$

which is the same dispersion relation as that derived by Chandrasekhar and Fermi [16]. In fact, the authors [16] used a totally different method compared to the one used here. 
They used the method of representing solenoidal vectors in terms of poloidal and toroidal quantities.

If we suppose that $\left(\alpha=0, H_{0}=0, U=0, W=0, G=0\right.$ and $\left.m=0\right)$, the relation (69) yields

$$
\sigma^{2}=\frac{T}{\rho R_{0}^{3}} \frac{x I_{1}(x)}{I_{0}(x)}\left(1-x^{2}\right)
$$

This relation coincides with that derived regarding the capillary instability of a full liquid jet in a vacuum by Rayleigh [1].

If we suppose that ( $\alpha=1, U=0, W=0, T=0$ and $m=0$ ), the relation (69) reduces to

$$
\sigma^{2}=4 \pi G \rho \frac{x I_{1}(x)}{I_{0}(x)}\left[I_{0}(x) K_{0}(x)-\frac{1}{2}\right]+\frac{\mu H_{0}^{2}}{\sigma R_{0}^{2}}\left[-x^{2}+\frac{x^{2} I_{1}(x) K_{0}(x)}{I_{0}(x)\left(-K_{1}(x)\right)}\right],
$$

from which we obtain

$$
\sigma^{2}=4 \pi G \rho \frac{x I_{1}(x)}{I_{0}(x)}\left[I_{0}(x) K_{0}(x)-\frac{1}{2}\right]+\frac{\mu H_{0}^{2}}{\rho R_{0}^{2}} \frac{x}{I_{0}(x) K_{1}(x)},
$$

where use has been made of the Wronskian

$$
I_{m}(x) K_{m}^{\prime}(x)-I_{m}^{\prime}(x) K_{m}(x)=-x^{-1}
$$

for $m=0$. The relation (73) was established by Chandrasekhar [5] for axisymmetric disturbances.

\section{Stability discussions}

\subsection{Capillary instability}

In the absence of the magnetic field, we assume that the streaming fluid is acted upon only by the capillary force. In such a case, the dispersion relation of this model is given from the relation (69) in the form

$$
(\sigma+i m W+i k U)^{2}=\frac{T}{\rho R_{0}^{3}} \frac{x I_{1}(x)}{I_{0}(x)}\left(1-m^{2}-x^{2}\right) .
$$

By using the fact, for each non-zero real value of $x$ and $m \geq 0$, that

$$
\begin{aligned}
& I_{m}(x)>0, \\
& I_{m}^{\prime}(x)>0,
\end{aligned}
$$

the analytical and numerical discussions of the relation (76) reveal the following results.

In the computer for different values of $M$ and different cases of $U^{*}$ and $W^{*}$.

In the most important sausage mode $m=0$.

The dimensionless dispersion relation is

$$
\frac{(\sigma+i m W+i k U)^{2}}{4 \pi G \rho}=\frac{x I_{m}^{\prime}(x)}{I_{m}(x)}\left[I_{m}(x) K_{m}(x)-\frac{1}{2}\right]+M\left[\left(1-m^{2}-x^{2}\right) \frac{x I_{m}^{\prime}(x)}{I_{m}(x)}\right],
$$




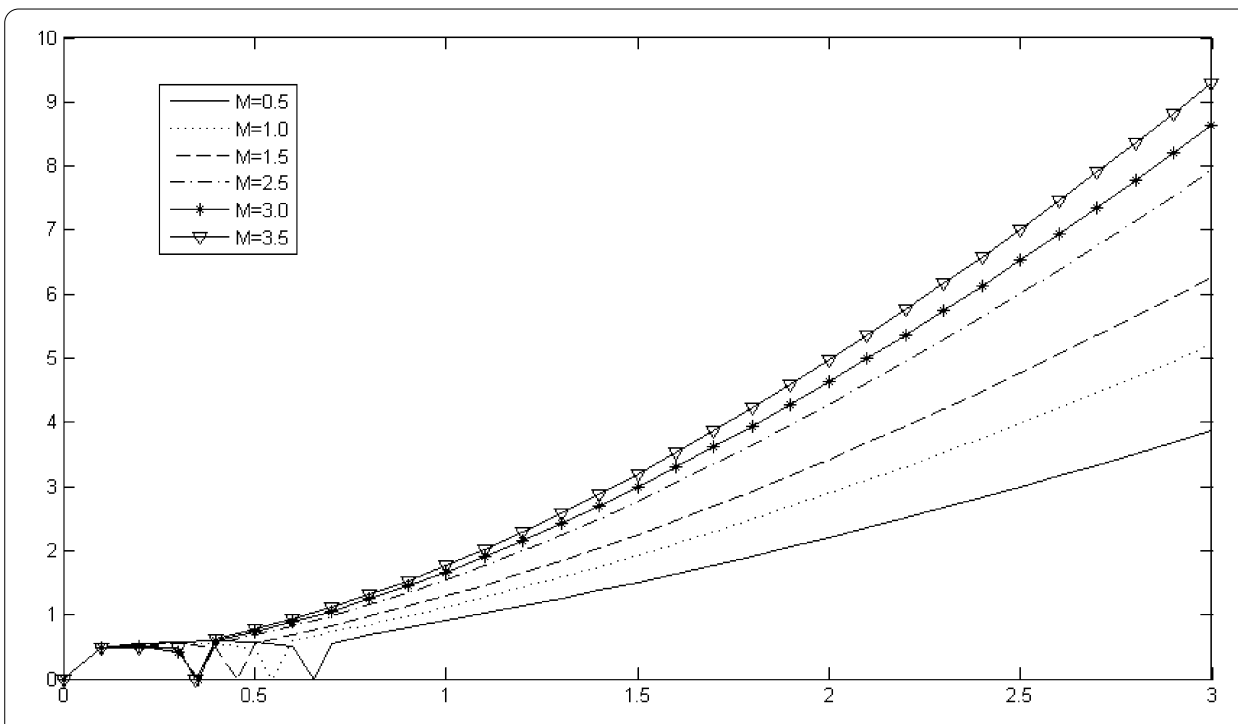

Figure 2 Stable and unstable domains for $\gamma=0, U^{*}=W^{*}=0.2$.

where

$$
M=\left[\frac{T}{\left(4 \pi G \rho^{2} R_{0}^{3}\right)}\right], \quad U^{*}=\left[\frac{-i k U}{(4 \pi G \rho)^{1 / 2}}\right], \quad W^{*}=\left[\frac{-i m W}{(4 \pi G \rho)^{1 / 2}}\right] .
$$

The numerical data associated with $\sigma /(4 \pi G \rho)^{\frac{1}{2}}$ correspond to the unstable states, while those associated with $\omega /(4 \pi G \rho)^{\frac{1}{2}}$ correspond to the stable domains. It has been found that there are many features of interest in this numerical analysis as we see in the following.

(i) For $M=0.5,1.0,1.5,2.5,3.0,3.5$, see Figure 2.

Corresponding to $U^{*}=W^{*}=0.2$. It has been found that the unstable domains are

$$
\begin{aligned}
& 0<x<0.65432, \quad 0<x<0.5451, \quad 0<x<0.4542 \text {, } \\
& 0<x<0.3541, \quad 0<x<0.3643, \quad \text { and } \quad 0<x<0.3752 \text {, }
\end{aligned}
$$

while the neighboring stable domains are

$$
\begin{aligned}
& 0.65432 \leq x<\infty, \quad 0.5451 \leq x<\infty, \quad 0.4542 \leq x<\infty, \\
& 0.3541 \leq x<\infty, \quad 0.3643 \leq x<\infty, \quad \text { and } \quad 0.3752 \leq x<\infty,
\end{aligned}
$$

where the equalities correspond to the marginal stability states.

(ii) For $M=0.5,1.0,1.5,2.5,3.0,3.5$, see Figure 3.

Corresponding to $U^{*}=W^{*}=0.5$. It has been found that the unstable domains are

$$
\begin{aligned}
& 0<x<0.6542, \quad 0<x<0.5451, \quad 0<x<0.4543, \\
& 0<x<0.3542, \quad 0<x<0.3455, \quad \text { and } \quad 0<x<0.3342 \text {, }
\end{aligned}
$$




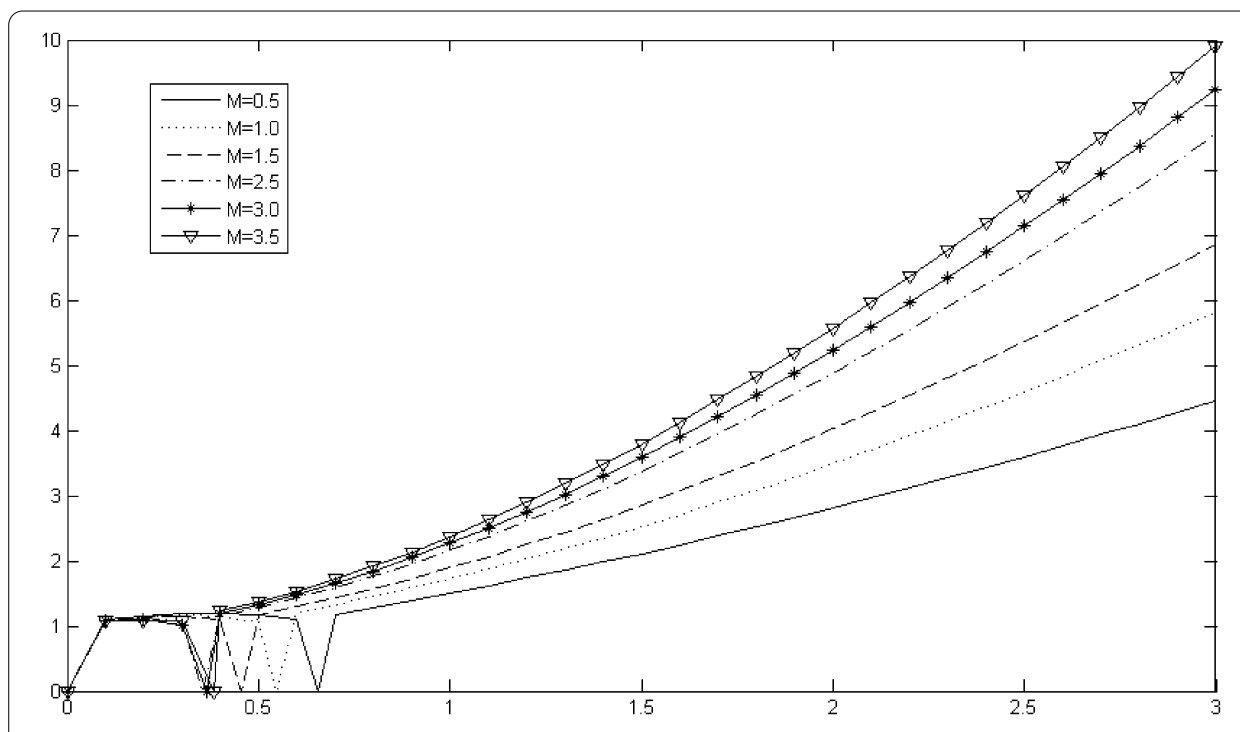

Figure 3 Stable and unstable domains for $\gamma=0, U^{*}=W^{*}=0.5$.

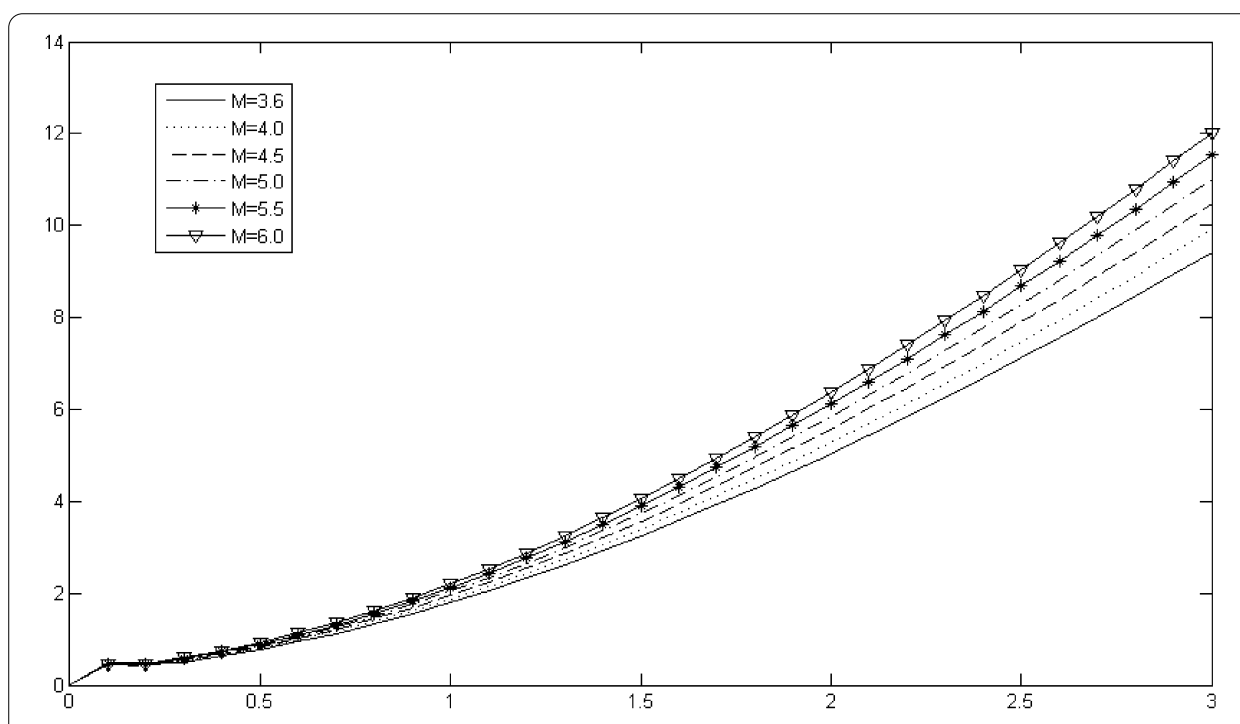

Figure 4 Stable domains for $\gamma=0, U^{*}=W^{*}=0.2$.

while the neighboring stable domains are

$$
\begin{aligned}
& 0.6542 \leq x<\infty, \quad 0.5451 \leq x<\infty, \quad 0.4543 \leq x<\infty, \\
& 0.3542 \leq x<\infty, \quad 0.3655 \leq x<\infty, \quad \text { and } \quad 0.3842 \leq x<\infty \text {, }
\end{aligned}
$$

where the equalities correspond to the marginal stability states.

(iii) For $M=3.6,4.0,4.5,5.0,5.5,6.0$, see Figure 4.

Corresponding to $U^{*}=W^{*}=0.2$. It has been found that stable domains are $0 \leq x<\infty$.

(iv) For $M=3.6,4.0,4.5,5.0,5.5,6.0$, see Figure 5.

Corresponding to $U^{*}=W^{*}=0.5$. It has been found that stable domains are $0 \leq x<\infty$. 


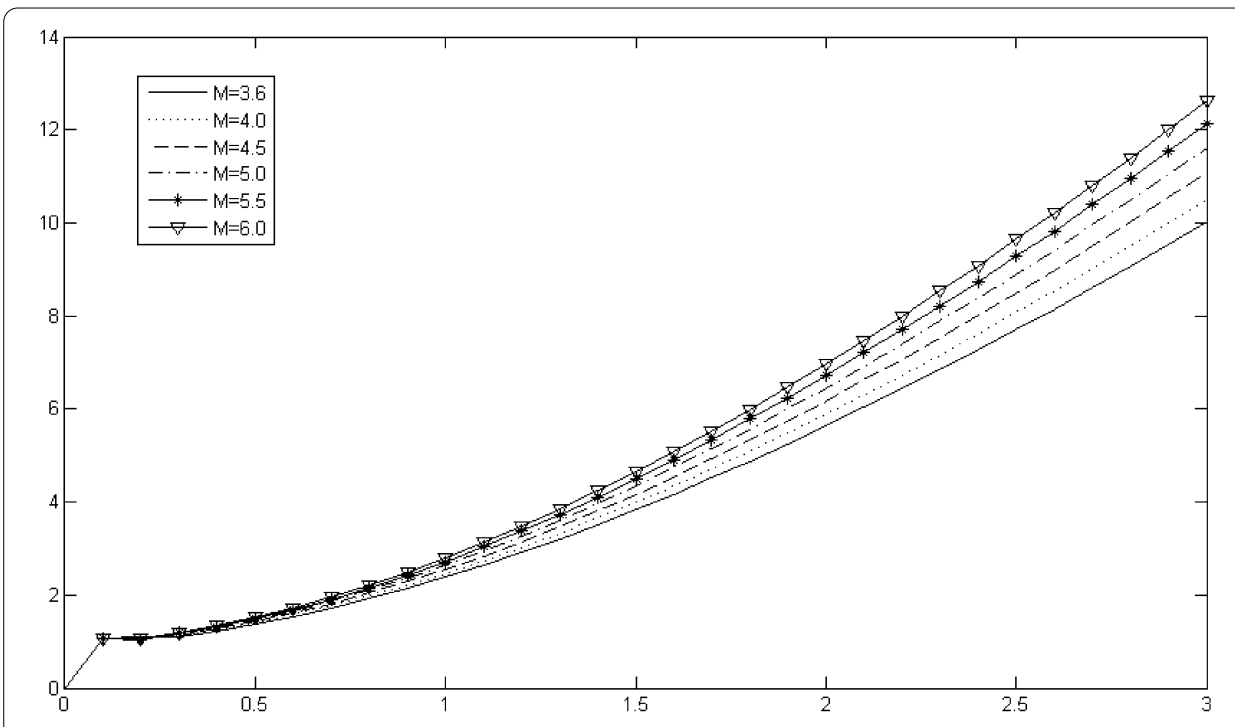

Figure 5 Stable domains for $\gamma=0, U^{*}=W^{*}=0.5$.

We conclude that the streaming full fluid cylinder has stable and unstable domain for $M$ less than 3.5 and stable domain only for $M$ greater than this value whatever the values of velocities are. Increasing the value of $M$, the unstable domain is decreasing. The effect of changing velocities cases on the capillarity effect is such small that it may be considered as no effect.

\subsection{Self-gravitating instability}

Consider only the self-gravitating force effect, and then the dispersion relation of the model is given from equation (69) as follows:

$$
(\sigma+i m W+i k U)^{2}=4 \pi G \rho \frac{x I_{m}^{\prime}(x)}{I_{m}(x)}\left[I_{m}(x) K_{m}(x)-\frac{1}{2}\right] .
$$

Consider the inequalities (77) and (78) and, for each non-zero real value of $x$, that

$$
K_{m}(x)>0
$$

the analytical and numerical discussion of the relation (79) reveal the following.

For $U=0, W=0$, it has been found that the model is gravitationally unstable in the domain $(0<x<1.0667$ for $m=0$ mode) while it is stable in the domains $(1.0667 \leq x \leq \infty$ for $m=0$ mode) and ( $0 \leq x \leq \infty$ for $m \geq 1$ modes).

For $U \neq 0, W \neq 0$, it has been found that the axial flow has a strong destabilizing influence. That effect does not rely on the kind of perturbation and it is so for all short and long wavelengths. Therefore, the streaming has the effect of increasing the axisymmetric stable domain $1.0667 \leq x \leq \infty$ and the non-axisymmetric domains $0<x<\infty$.

We conclude that the streaming self-gravitating fluid cylinder is unstable not only for the axisymmetric mode $m=0$, but also for non-axisymmetric modes $m \geq 1$. 


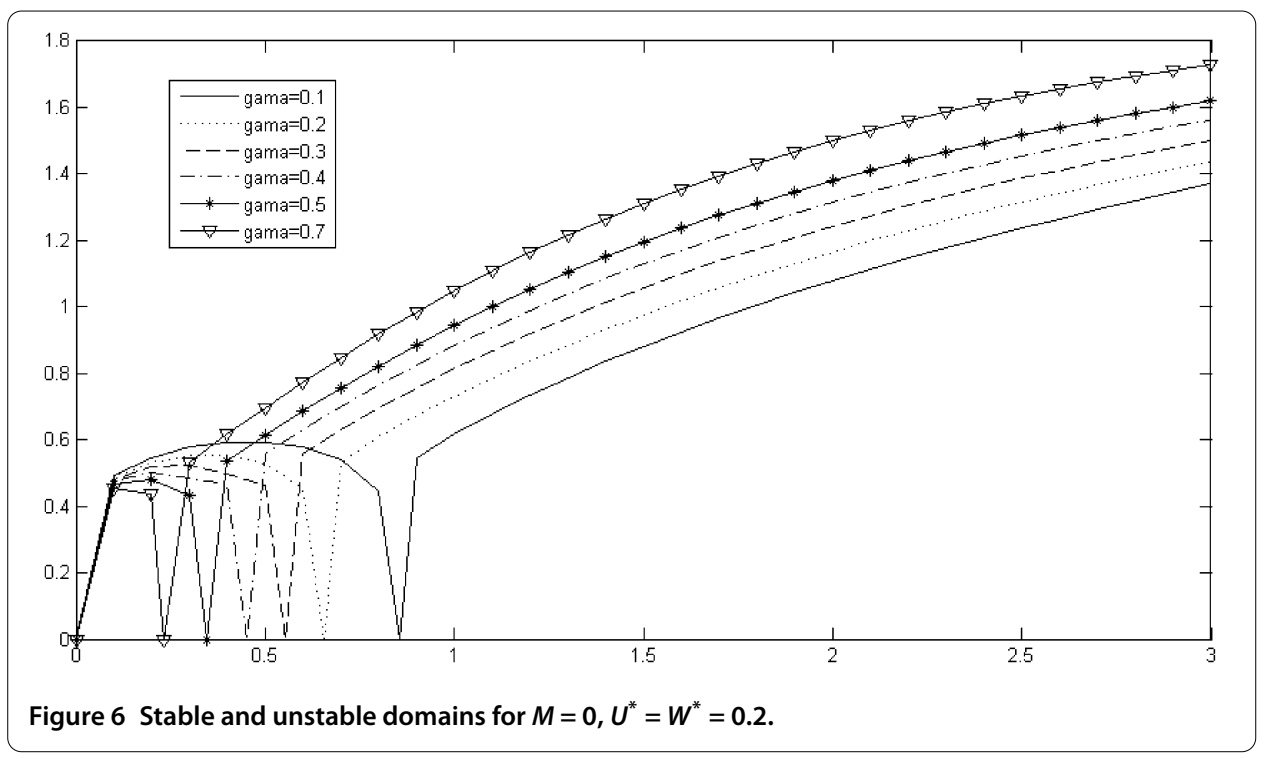

\subsection{Magnetogravitodynamic stability}

This is the case in which the streaming fluid cylinder is acted upon by the combined effects of the self-gravitating and magnetic forces. It is difficult to determine exactly in analytical ways the (un-) stable domains in such a general case. However, we could determine them via the numerical discussions. Also, by means of such discussion, we may find out the effects of the magnetic field on the self-gravitating force. This could be carried out by calculating the dimensionless dispersion relation

$$
\frac{(\sigma+i m W+i k U)^{2}}{4 \pi G \rho}=\frac{x I_{m}^{\prime}(x)}{I_{m}(x)}\left[I_{m}(x) K_{m}(x)-\frac{1}{2}\right]+\gamma\left[-x^{2}+\alpha^{2} \frac{x^{2} I_{m}^{\prime}(x) K_{m}(x)}{I_{m}(x) K_{m}^{\prime}(x)}\right]
$$

in the computer for different values of

$$
\gamma\left(=\left(\mu / 16 \pi^{2} G\right)\left(H_{0} / \rho R_{0}\right)^{2}\right) \quad \text { and } \quad U^{*}=\left[\frac{-i k U}{(4 \pi G \rho)^{1 / 2}}\right]
$$

in the most important sausage mode $m=0$.

The numerical data associated with $\sigma /(4 \pi G \rho)^{\frac{1}{2}}$ correspond to the unstable states, while those associated with $\omega /(4 \pi G \rho)^{\frac{1}{2}}$ correspond to the stable domains. It has been found that there are many features of interest in this numerical analysis as we see in the following.

(i) For $\gamma=0.1,0.2,0.3,0.4,0.5,0.7$, see Figure 6 .

Corresponding to $U^{*}=W^{*}=0.2$. It has been found that the unstable domains are

$$
\begin{aligned}
& 0<x<0.8543, \quad 0<x<0.65423, \quad 0<x<0.5541, \\
& 0<x<0.45323, \quad 0<x<0.3452, \quad \text { and } \quad 0<x<0.2342,
\end{aligned}
$$

while the neighboring stable domains are

$$
\begin{aligned}
& 0.8543 \leq x<\infty, \quad 0.65423 \leq x<\infty, \quad 0.65423 \leq x<\infty, \\
& 0.65423 \leq x<\infty, \quad 0.65423 \leq x<\infty, \quad \text { and } \quad 0.65423 \leq x<\infty,
\end{aligned}
$$

where the equalities correspond to the marginal stability states. 


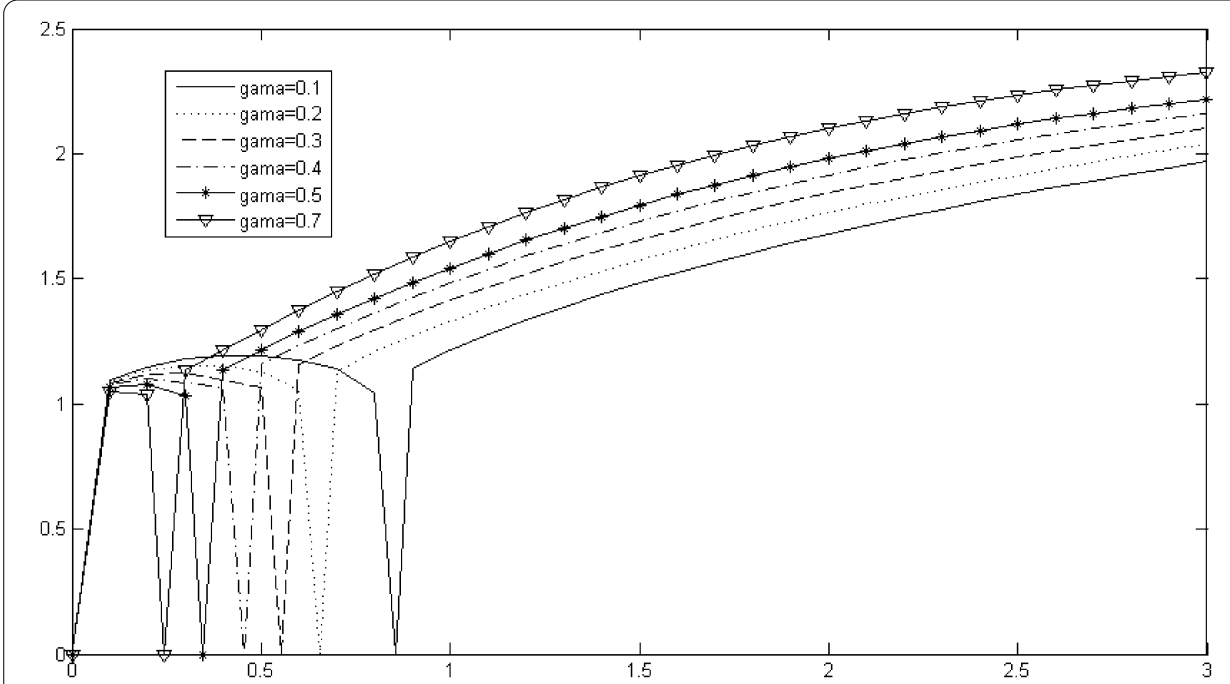

Figure 7 Stable and unstable domains for $M=0, U^{*}=W^{*}=0.5$.

(ii) For $\gamma=0.1,0.2,0.3,0.4,0.5,0.7$, see Figure 7 .

Corresponding to $U^{*}=W^{*}=0.5$. It has been found that the unstable domains are

$$
\begin{aligned}
& 0<x<0.87412, \quad 0<x<0.6742, \quad 0<x<0.5841, \\
& 0<x<0.4743, \quad 0<x<0.3754, \quad \text { and } \quad 0<x<0.2456,
\end{aligned}
$$

while the neighboring stable domains are

$$
\begin{aligned}
& 0.85412 \leq x<\infty, \quad 0.6542 \leq x<\infty, \quad 0.5541 \leq x<\infty, \\
& 0.4543 \leq x<\infty, \quad 0.3454 \leq x<\infty, \quad \text { and } \quad 0.2456 \leq x<\infty,
\end{aligned}
$$

where the equalities correspond to the marginal stability states.

(iii) For $\gamma=0.8,0.9,1.0,1.2,1.3,1.4$, see Figure 8.

Corresponding to $U^{*}=W^{*}=0.2$. It has been found that stable domains are $0 \leq x<\infty$.

(iv) For $\gamma=0.8,0.9,1.0,1.2,1.3,1.4$, see Figure 9.

Corresponding to $U^{*}=W^{*}=0.5$. It has been found that stable domains are $0 \leq x<\infty$.

We conclude that the streaming full fluid cylinder has stable and unstable domain for $\gamma$ less than 0.8 and stable domain only. Increasing the value of magnetic field, the unstable domains are decreasing. The effect of changing velocities cases on magnetic effect is such small that it may be considered asno effect. If we compare these results with those of chapter two (only velocity in $z$ direction), we observe that the existance of another velocity $W$ in $\varphi$ direction decreases the unstable domain.

\subsection{Magnetogravitodynamic capillary stability}

This is the general case in which the streaming fluid cylinder is acted upon by the combined effects of the self-gravitating, capillary, and magnetic forces. The dispersion relation is given in its general form by equation (69). It is difficult to determine exactly in analytical 

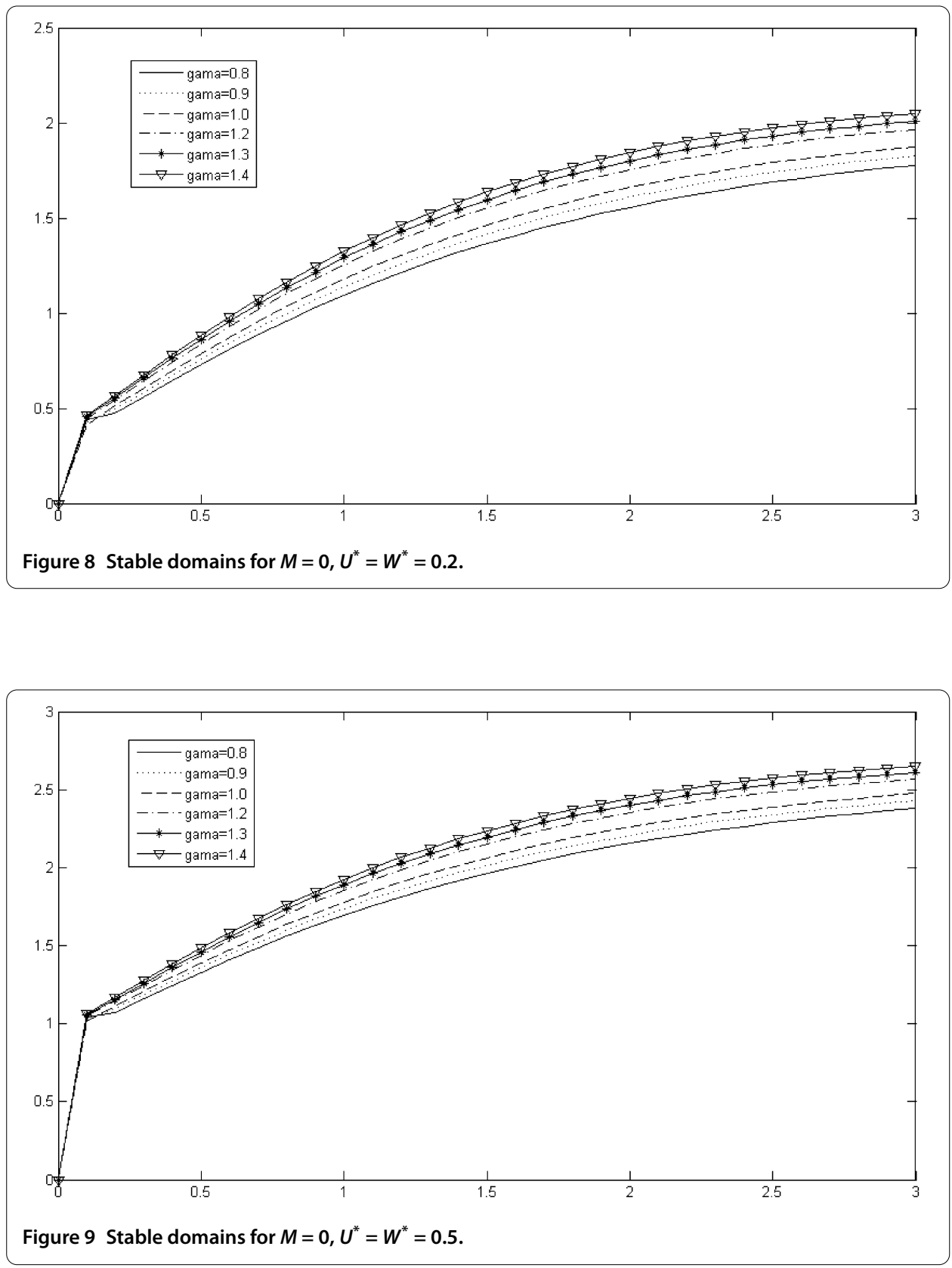

ways the (un-) stable domains in such a general case. However, we could determine them via the numerical discussions. Also, by means of such discussion, we may find out the effects of capillary with a constant magnetic field on the self-gravitating force. This could be carried out by calculating the dimensionless dispersion relation

$$
\begin{aligned}
\frac{(\sigma+i m W+i k U)^{2}}{4 \pi G \rho}= & \frac{x I_{m}^{\prime}(x)}{I_{m}(x)}\left[I_{m}(x) K_{m}(x)-\frac{1}{2}\right]+M\left[\left(1-m^{2}-x^{2}\right) \frac{x I_{m}^{\prime}(x)}{I_{m}(x)}\right] \\
& +\gamma\left[-x^{2}+\alpha^{2} \frac{x^{2} I_{m}^{\prime}(x) K_{m}(x)}{I_{m}(x) K_{m}^{\prime}(x)}\right]
\end{aligned}
$$




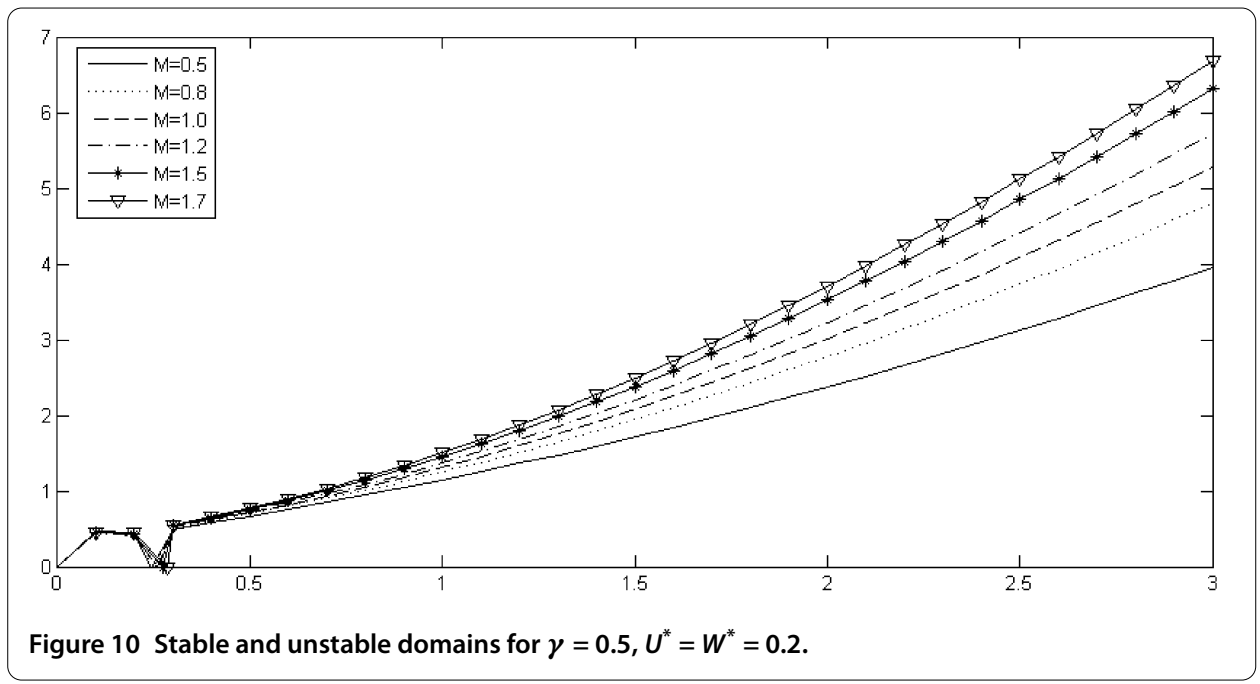

in the computer for different values of

$$
M=\left[\frac{T}{\left(4 \pi G \rho^{2} R_{0}^{3}\right)}\right], \quad \gamma\left(=\left(\mu / 16 \pi^{2} G\right)\left(H_{0} / \rho R_{0}\right)^{2}\right) \quad \text { and } \quad U^{*}=\left[\frac{-i k U}{(4 \pi G \rho)^{1 / 2}}\right]
$$

in the most important sausage mode $m=0$.

The numerical data associated with $\sigma /(4 \pi G \rho)^{\frac{1}{2}}$ correspond to the unstable states, while those associated with $\omega /(4 \pi G \rho)^{\frac{1}{2}}$ correspond to the stable domains. It has been found that there are many features of interest in this numerical analysis as we see in the following.

(i) For $M=0.5,1.0,1.5,2.5,3.0,3.5$, see Figure 10 .

Corresponding to $\gamma=0.5$ and $U^{*}=W^{\prime \prime}=0.2$. It has been found that the unstable domains are

$$
\begin{aligned}
& 0<x<0.24321, \quad 0<x<0.2523, \\
& 0<x<0.2641, \quad 0<x<0.25743,
\end{aligned}
$$

while the neighboring stable domains are

$$
\begin{aligned}
& 0.24321 \leq x<\infty, \quad 0.2523 \leq x<\infty, \quad 0.25743 \leq x<\infty, \\
& 0.2641 \leq x<\infty, \quad 0.2732 \leq x<\infty, \quad \text { and } \quad 0.2843 \leq x<\infty,
\end{aligned}
$$

where the equalities correspond to the marginal stability states.

(ii) For $M=0.5,1.0,1.5,2.5,3.0,3.5$, see Figure 11 .

Corresponding to $\gamma=0.5$ and $U^{*}=W^{\prime \prime}=0.5$. It has been found that the unstable domains are

$$
\begin{aligned}
& 0<x<0.2341, \quad 0<x<0.2532, \quad 0<x<0.2573, \\
& 0<x<0.2764, \quad 0<x<0.2825, \text { and } 0<x<0.2944 \text {, }
\end{aligned}
$$




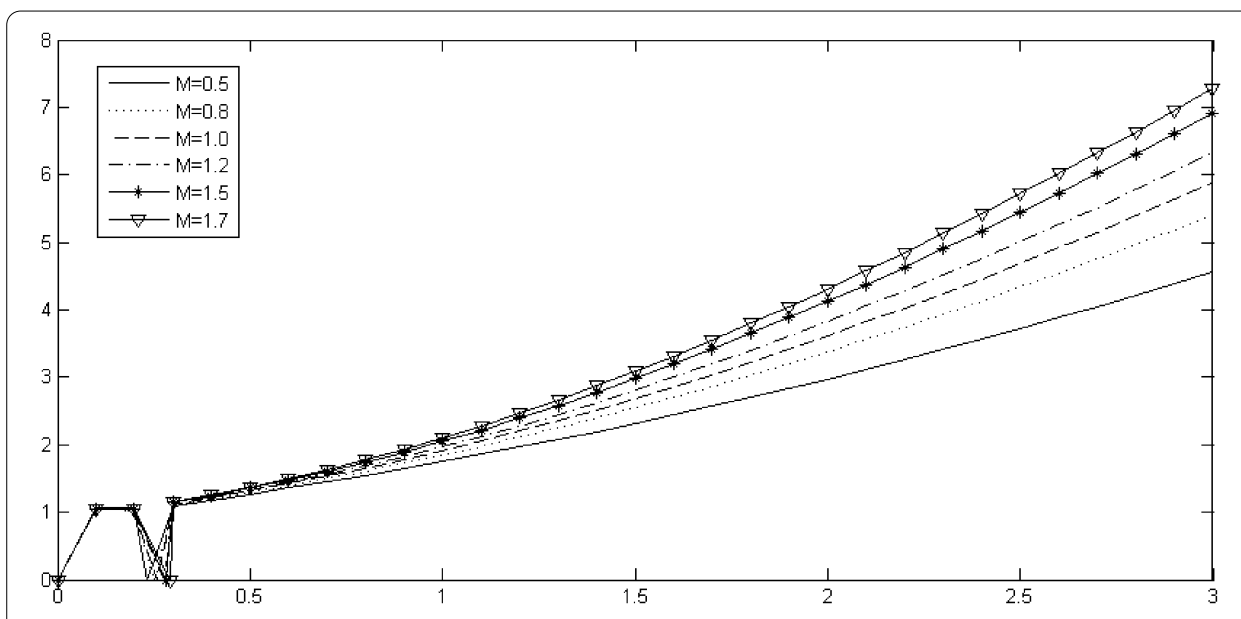

Figure 11 Stable and unstable domains for $\gamma=0.5, U^{*}=W^{*}=0.5$.

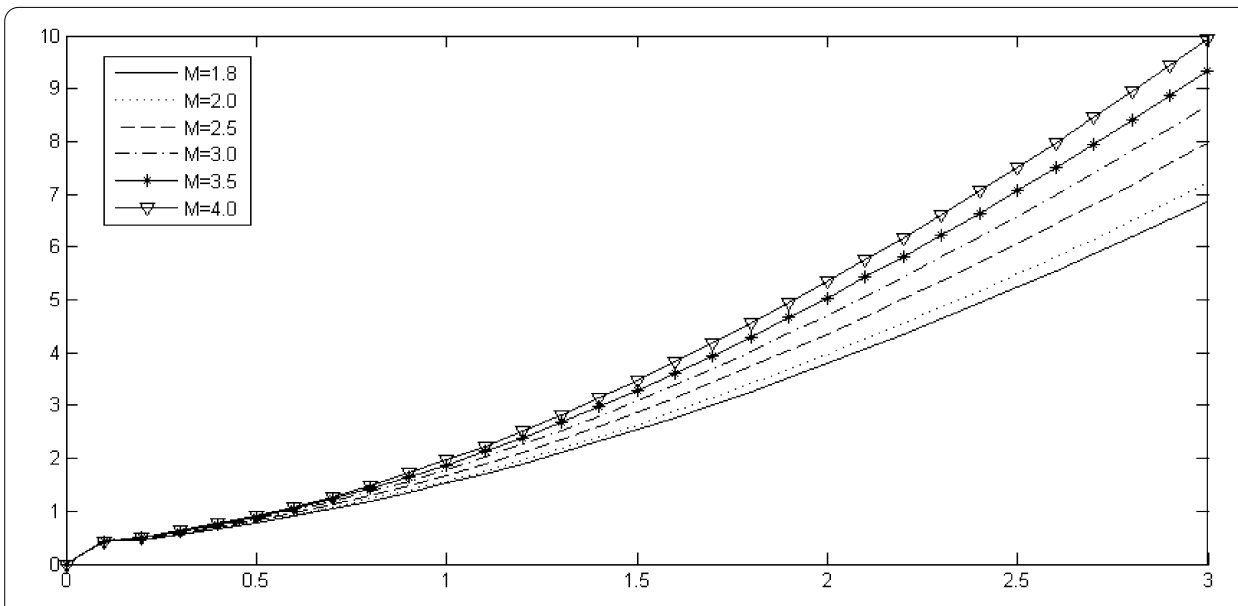

Figure 12 Stable domains for $\gamma=0.5, U^{*}=W^{*}=0.2$.

while the neighboring stable domains are

$$
\begin{array}{ll}
0.2341 \leq x<\infty, & 0.2532 \leq x<\infty, \quad 0.2573 \leq x<\infty, \\
0.2764 \leq x<\infty, & 0.2825 \leq x<\infty, \quad \text { and } \quad 0.2944 \leq x<\infty,
\end{array}
$$

where the equalities correspond to the marginal stability states.

(iii) For $M=0.5,1.0,1.5,2.5,3.0,3.5$, see Figure 12 .

Corresponding to $\gamma=0.5$ and $U^{*}=W^{*}=0.2$. It has been found that stable domains are

$$
0 \leq x<\infty
$$

(iv) For $M=0.5,1.0,1.5,2.5,3.0,3.5$, see Figure 13 .

Corresponding to $\gamma=0.5$ and $U^{\prime \prime}=W^{*}=0.5$. It has been found that stable domains are

$$
0 \leq x<\infty
$$




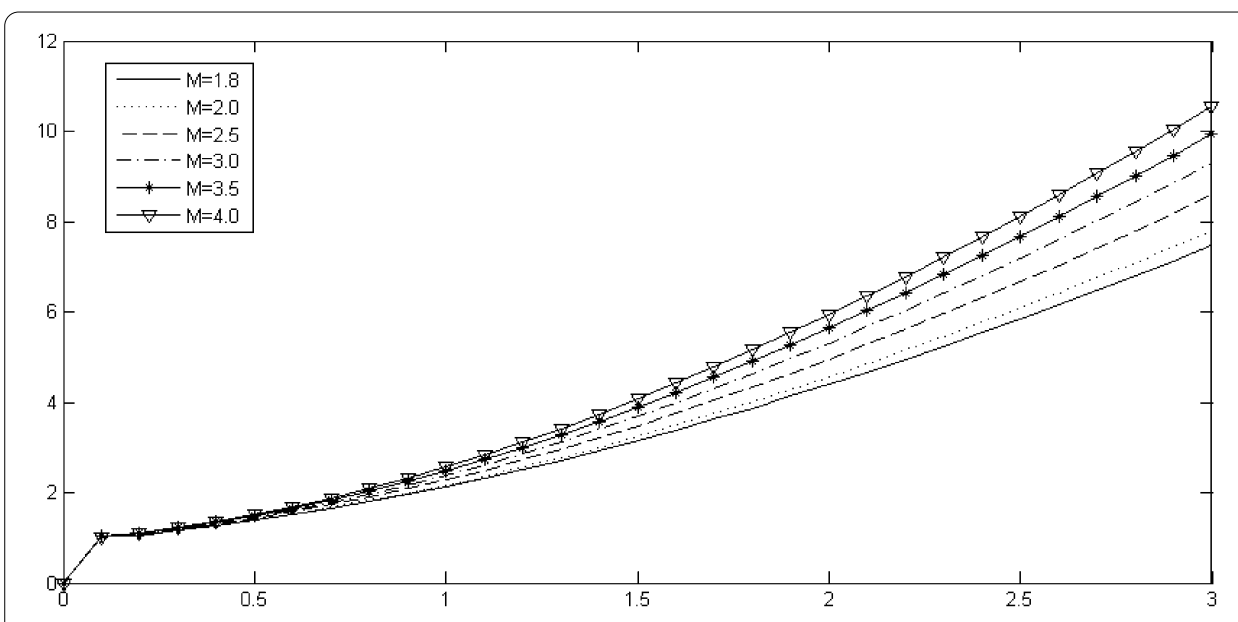

Figure 13 Stable domains for $\gamma=0.5, U^{*}=W^{*}=0.5$.

We conclude that the streaming full fluid cylinder has stable and unstable domains for $M$ less than 3.5 and stable domain only for $M$ greater than this value whatever the values of velocities are. The effect of changing velocities cases on capillarity effect is such small that it may be considered as no effect. Increasing $M$ with constant magnetic field increases the unstable domain.

\section{Conclusion}

From the foregoing numerical results, we may deduce the following:

(1) The velocity has a strong destabilizing influence on the self-gravitating instability of the model.

(2) The capillary force has a strong stabilizing influence on the self-gravitating instability of the model.

(3) The capillary and self-gravitating modified a lot the instability of the model for all short and long wavelengths.

(4) The velocity has a strong destabilizing influence on the self-gravitating instability of the model.

(5) The magnetic force has a strong stabilizing influence on the self-gravitating instability of the model.

(6) The self-gravitating instability character has disappeared and has been dispersed, and the model has become completely stable.

(7) The velocities in two directions have a strong destabilizing influence on the self-gravitating instability of the model.

(8) The magnetic force has a strong stabilizing influence on the self-gravitating capillary instability of the model. 


\section{Author details}

${ }^{1}$ Basic and Applied Sciences Department, College of Engineering and Technology, Arab Academy for Science \& Technology and Maritime Transport (AASTMT), Elhorria, P.O. Box 2033, Cairo, Egypt. ${ }^{2}$ Physics and Engineering Mathematics Department, Faculty of Engineering (Mattria), Helwan University, Cairo, Egypt.

\section{Acknowledgements}

We are grateful to the editor of the journal and the reviewers for their suggestion and comments of this paper.

\section{Received: 23 October 2012 Accepted: 15 February 2013 Published: 7 March 2013}

\section{References}

1. Rayleigh, JM: The Theory of Sound. Dover, New York (1945)

2. Yuen, MC: Non-linear capillary instability of a liquid jet. J. Fluid Mech. 33, 151 (1968)

3. Nayfeh, A, Hassan, SD: The method of multiple scales and non-linear dispersive waves. J. Fluid Mech. 48, 463 (1971)

4. Kakutani, T, Inoue, I, Kan, T: Nonlinear capillary waves on the surface of liquid column. J. Phys. Soc. Jpn. 37, 529 (1974)

5. Chandrasekhar, S: Hydrodynamic and Hydromagnetic Stability. Dover, New York (1981)

6. Radwan, AE, Aly, FA: Selfgravitating instability of two semi-infinite streaming superposed fluids endowed with surface tension. Nuovo Cimento 113, 601 (1998)

7. Radwan, AE, Ali, RM: Magnetohydrodynamic instability of a dissipative compressible rotating selfgravitating fluid medium. Nuovo Cimento 114B, 1361 (1999)

8. Radwan, AE: Periodic time dependent electrogravitational instability of a fluid cylinder. Phys. Scr. 67, 510 (2007)

9. Radwan, AE, Hasan, AA: Axisymmetric electrogravitational stability of fluid cylinder ambient with transverse varying oscillating field. Int. J. Appl. Math. 38(3), 13 (2008)

10. Radwan, AE, Hasan, AA: Magnetohydrodynamic stability of self-gravitational fluid cylinder. Appl. Math. Model. 33, 2121 (2009)

11. Hasan, AA: Electrogravitational stability of oscillating streaming fluid cylinder. Physica B 406(2), 234 (2011)

12. Hasan, AA: Capillary electrodynamic stability of self-gravitational fluid cylinder with varying electric field. J. Appl. Mech. 79(2), 1 (2011)

13. Hasan, AA, Mekheimer, KS, Azwaz, SL: Hydromagnetic stability of selfgravitational oscillating streaming fluid jet pervaded by azimuthal varying magnetic field. Int. J. Math. Arch. 2(4), 488 (2011)

14. Hasan, AA: Electrogravitational stability of oscillating streaming dielectric compound jets ambient with a transverse varying electric field. Bound. Value Probl. 2011, 31 (2011)

15. Hasan, AA: Hydromagnetic instability of streaming jet pervaded internally by varying transverse magnetic field. Math Probl. Eng. 2012, 325423 (2012)

16. Chandrasekhar, S, Fermi, E: Problems of gravitational stability in the presence of a magnetic field. Astrophys. J. 118, 116 (1953)

\section{Submit your manuscript to a SpringerOpen ${ }^{\mathcal{D}}$ journal and benefit from:}

- Convenient online submission

Rigorous peer review

- Immediate publication on acceptance

- Open access: articles freely available online

- High visibility within the field

- Retaining the copyright to your article 